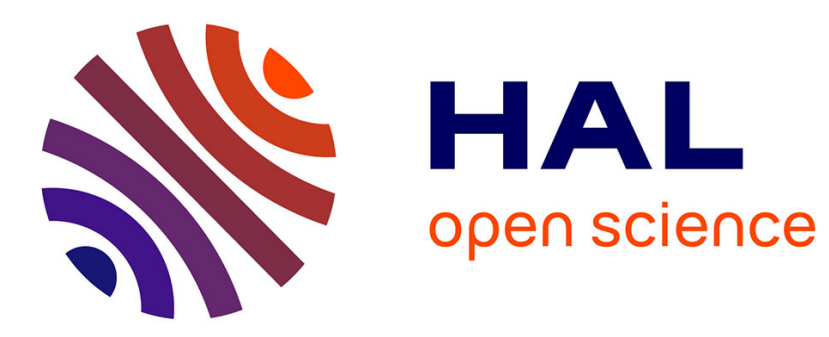

\title{
La diplomatie publique chinoise sur Twitter : la fabrique d'une polyphonie harmonieuse
}

Zhao Alexandre Huang, Olivier Arifon

\section{To cite this version:}

Zhao Alexandre Huang, Olivier Arifon. La diplomatie publique chinoise sur Twitter: la fabrique d'une polyphonie harmonieuse. Hermès, La Revue - Cognition, communication, politique, 2018, 2 (81), pp.45-53. 10.3917/herm.081.0045 . hal-02106328

\section{HAL Id: hal-02106328 \\ https://hal.science/hal-02106328}

Submitted on 22 Apr 2019

HAL is a multi-disciplinary open access archive for the deposit and dissemination of scientific research documents, whether they are published or not. The documents may come from teaching and research institutions in France or abroad, or from public or private research centers.
L'archive ouverte pluridisciplinaire HAL, est destinée au dépôt et à la diffusion de documents scientifiques de niveau recherche, publiés ou non, émanant des établissements d'enseignement et de recherche français ou étrangers, des laboratoires publics ou privés. 


\section{« Hermès : La Revue »}

\section{Communications et incommunications diplomatiques}

Auteurs :

Zhao Huang est doctorant contractuel avec mission d'enseignement en Sciences de l'Information et de la Communication à la COMUE Université Paris-Est. Rattaché au laboratoire DICEN-IDF, sa thèse porte sur la diplomatie publique de la Chine en Afrique. Ancien journaliste à la Radio Nationale de Chine, il étudie les pratiques instituantes, les stratégies de communication politique et publique, et la narration stratégique dans le processus de la diplomatie publique.

Olivier Arifon est enseignant-chercheur. Entre 2011 et 2014, il était professeur en Sciences de l'information et de la communication à l'Université libre de Bruxelles. Depuis 20 ans, au sein des SIC, il enseigne l'intelligence économique, la diplomatie publique et le lobbying. Ses recherches portent sur la communication, le lobbying et les sociétés civiles dans une perspective comparée entre Chine, Inde et Europe.

Contacts : zhao.huang@u-pem.fr ; oarifon@ulb.ac.be;

Titre : Diplomatie publique et voix diplomatiques chinoises à Bruxelles et Ottawa sur Twitter : la fabrique d'une polyphonie harmonieuse

Mots-clés : Chine, diplomatie publique, diplomatie digitale, Twiplomatie, infomédiation, polyphonie 


\section{Introduction}

Le « rêve chinois », "wai xuan », est un thème central dans la propagande chinoise et la communication diplomatique de Pékin. Cette notion de « Rêve chinois » ou « Rêve de la Chine » a été lancée par le président Xi Jinping pour désigner ses objectifs politiques durant son mandat présidentiel. Selon le politologue chinois Lanxin Xiang, ce slogan politique se décompose en trois objectifs : « restaurer la gloire passée de la Chine et de l'État; rappeler le désir séculaire d'une Chine moderne, riche et puissante, qu'ont eu tous les empereurs ; et rendre les Chinois fiers et heureux, afin de maintenir la stabilité sociale » (Le Belzic, 2013). La polysémie de cette expression frappante, la Chine peut être envisagée comme le sujet rêvant et comme l'objet du rêve, une Chine que les citoyens nationaux, mais aussi les dirigeants et les peuples du monde entier doivent rêver, au sens d'idéaliser. Alors que la propagande est associée dans les esprits des publics français et occidentaux à une forme de communication politique problématique en termes d'éthique puisqu'elle intègre dans ses discours des éléments de désinformation (Ollivier-Yaniv, 2010), les chercheurs chinois reconnaissent des vertus à la propagande, et «une connotation positive associée à ses activités communicationnelles essentiellement douces ou bégnines telles que la publication de nouvelles, la fabrication et la pédagogie idéologique ou même la publicité 》 (Wang, 2008, p. 259). Certains chercheurs chinois considèrent la diplomatie publique comme le prolongement de la propagande intérieure, en ce qu'elle se déploie à travers de nombreuses formes discursives et plateformes médiatiques, et puisqu'elle vise la promotion de la Chine auprès des publics étrangers (Chen et Liu, 2015; Zhou, 2010).

Si la propagance est une forme très hiérarchisée, centralisée et unilatérale de communication, les activités relavant de la diplomatie publique sont polyphoniques car nombreuses sont les voix de la Chine s'exprimer à l'étranger, mais elles sont aussi interactives dans la mesure où de nombreux publics libres d'expression peuvent par exemple commentés les publications des diplomates chinois sur leurs comptes sur les médias socionumériques. Du fait des risques de moindre maitrise du discours et de cacophonie communicationnelle liés à la multiplicité des voix (Arsène, 2012; Li, 2015), la Chine a commencé prudemment à inviter ses diplomates à s'exprimer officiellement sur Twitter, et le gouvernement ne gère que huit comptes diplomatiques sur Twitter. De plus, les missions de « wai xuan », et donc de promotion du « rêve chinois », sont dirigées par le Département de la Propagande du Parti communiste de Chine, elles sont par conséquent fortement encadrées et contrôlées. On parle à ce sujet en Chine «d'actions prescrites ». Dans ce contexte si particulier de communication sous contrainte forte et d'éthos diplomatique fait de prudence, comment les diplomates chinois utilisent-ils le Twitter pour servir à la diplomatie publique de l'État ? Comment la culture érudite et le poids de l'histoire longue de la diplomatie s'accommodent-ils de médias sociaux marqués par le pluralisme de l'information, la brièveté des messages, l'extraordinaire diffusion vers tous les types de publics, la culture de l'instantanéité des commentaires et des réponses, la prédilection pour les slogans (Lafrance, $2011, \S 4)$ et pour l'humour? Comment les diplomates chinois en poste à l'étranger qui alimentent des comptes officiels parviennent à exprimer des voix différentes ou personnelles, mais harmonieuses dans le respect des prescriptions gouvernementales ? Le cadre théorique mobilise pour répondre à ces questions associé les sciences politiques et les sciences de 
l'information et de la communication telles qu'elles sont développées partout dans le monde. Ainsi, nous avons voulu éclairer la manière dont les chercheurs chinois pensent la diplomatie publique et la diplomatie digitale dans une optique de soft power.

Le concept de diplomatie publique est fréquemment explicité dans les manuels de Science politique et de relations internationales, on le trouve aussi parfois dans des articles de presse et des discours politiques. Pour autant, l'effort de conceptualisation achoppe sur la diversité des pratiques supposément recouvertes par ce terme, ainsi que sur leur dimension idéologique, voire polémique. La définition la plus souvent citée dans les ouvrages anglosaxons et français envisage la diplomatie publique comme une activité diplomatique relevant de la communication stratégique en ce qu'elle vise à influer les cadres de pensées de très nombreuses catégories de publics étrangers et, en fin de compte à peser sur les processus de décision politiques de leurs gouvernements (Malone, 1985, p. 199). Les tenants de l'approche cognitive des politiques publiques parlent à ce sujet d'une recherche de mise sur agenda et d'un effort de cadrage des "référentiels », en tant qu' " ensemble de représentations qu'une société se donne pour comprendre et agir sur le réel» (Muller, 2014, p.555), et « processus cognitif fondant un diagnostic (Ibid., p.556). Si la définition de Malone insiste sur les finalités stratégiques de la diplomatie publique, Tuch précise quels peuvent en être les acteurs et contenus : «la communication effectuée par un gouvernement auprès de publics étrangers afin de promouvoir (une meilleure compréhension de) ses idées et idéaux, ses institutions et sa culture, ainsi que ses politiques et objectifs nationaux» (Tuch, 1990, p. 3). La diplomatie publique rassemble un ensemble de discours et de pratiques par lesquels les États cherchent à influencer des opinions publiques étrangères d'une manière discrète ou secrète sur des questions précises, en même temps qu'à améliorer l'image de leur État-nation sur la scène internationale, ce qui suppose de développer leurs échanges transculturels. La diplomatie publique est analysée par les tenants d'une conception patriotique de l'intelligence économique comme « une forme de communication impliquant des stratégies et tactiques de relations publiques afin de provoquer un changement d'attitudes et de dispositions chez les publics étrangers » (Zhong et Lu, 2013, p.543).

Les médias numériques, l'Internet, et les médias sociaux ont profondément impacté les pratiques de diplomatie publique en ce qu'elles sont tournées vers une très grande diversité de publics. L'utilisation des médias sociaux, par exemple, a réduit le coût de la mise en œuvre de stratégies de diplomatie publique en même temps qu'elle a permis de dynamiser les interactions entre les instances diplomatiques officielles et leurs publics. Ainsi, «la connectivité et l'interactivité sont les caractéristiques déterminantes des médias sociaux et l'activité communicationnelle qui définit ses acteurs » (Zaharna, 2010, p. 87). Dans la lignée des approches théoriques qui valorisent la co-création de sens à l'œuvre dans la communication (Weick, 1995 ; Botan et Taylor, 2004, p. 652), des chercheurs chinois considèrent que : «toutes les activités communicationnelles de la Chine, État-nation sur la scène internationale s'ancrent dans la vie sociale des pays et, visent autant qu'elles interagissent directement avec certains publics étrangers » (Chen et Liu, 2015, p. 25).

À partir d'une enquête qualitative approfondie des voix diplomatiques chinoises à Bruxelles et Ottawa étudiées dans leur environnement social et rapportée à l'actualité sur la scène politique internationale, cet article se propose de montrer que le gouvernement chinois reste prudent dans son utilisation diplomatique des médias sociaux, car le parti-État considère 
l'Internet comme une arme à double tranchant du fait des limites propres aux stratégies de censure (Arsène, 2009). «Avec Internet, le régime chinois fait du réseau une autre muraille constituée de multiples dispositifs de contrôle : contrôle technique, régulation juridique, pressions sociales, entreprises nationales et enfin tentative à l'international paragraphe » (Arifon, 2012, p.160). Le Parti communiste chinois exerce en effet une censure rigoureuse sur l'opinion publique de ses nationaux, tandis que les activités qui relèvent de la diplomatie publique sont contrôlées par le département de la propagande du Comité central du Parti communiste. Dans un contexte expressif marqué par le poids des «prescriptions » émanent du Parti unique, un nombre limité de personnes et institutions s'expriment sur les médias sociaux au nom de la diplomatie chinoise. Pourtant; leurs publications parviennent à créer une impression polyphonique auprès de ses publics mondiaux, une polyphonie harmonieuse qui entend « raconter des histoires à la chinoise et faire entendre la voix chinoise ${ }^{1}$.

\section{Une monophonie expressive incorporée dans un pluralisme des registres et des genres de discours}

Une grande homogénéité se dessine à travers le corpus recueilli de 1.592 tweets ce qui est cohérent avec leur mission officielle de représentation diplomatique ainsi qu'avec l'homogénéité sociologique et idéologique de leurs auteurs. Pour autant, les messages portent sur des sujets, emploient des registres et des styles de discours variés ce qui vient composer une impression polyphonique qui joue un rôle stratégique. Certains messages relèvent de la communication publique et politique, ils expriment et défendent les grandes lignes de la politique étrangère de la Chine. D'autres ne peuvent pas être rattachés à un élément précis de la politique chinoise, mais ils instruisent et plaident l'idée d'une Chine puissante, actrice incontournable et de premier plan dans le concert des nations. Enfin, des messages relèvent d'une stratégie de soft power.

\section{Exprimer et défendre les grandes lignes de la politique étrangère de la Chine}

Dès la réforme de 1978, le Parti communiste a tenté de défendre la légitimité de son rôle en tant que dirigeant du gouvernement central du pays (D’Hooghe, 2005, p. 93). Ce discours a pris une forme didactique et affective, il s'agissait pour le parti de gouvernement de saturer sa diplomatie publique d'explications au sujet de son mode de gouvernance très spécifique induit par la taille de sa population, sa géographie et son régime politique. En effet, 457 tweets de diplomates chinois illustrent et expliquent des politiques publiques chinoises très variées, mais aussi ce qui est décrit comme des « avantages institutionnels ». Ces messages sont très homogènes, notamment parce qu'ils citent de manière fidèle des extraits de discours d'hommes politiques ou d'interviews publiées ou prononcées dans les médias.

Les points saillants du discours du président Xi Jinping sont par exemple résumés dans un tweet publié par le compte China's mission to the European Union (@ChinaEUMission) le $1^{\text {er }}$ décembre 2017: «la Chine n'exportera pas le modèle chinois aux étrangers, ni

\footnotetext{
${ }^{1}$ Comme tous les messages twitters en anglais, cette phrase a été traduite du chinois par nos soins. Elle est issue du discours de Xi Jinping, président de la République populaire de Chine et Secrétaire général du Parti communiste chinois, prononcé durant la Conférence du Parti communiste chinois sur la Propagande et l'Idéologie en octobre 2013. Il développa dans ce discours sa vision innovante de la communication de la Chine sur la scène internationale. Il lui importait de développer des nouvelles formes d'expression tournées à la fois vers des publics domestiques et étrangers.
} 
"n’importera" des modèles étrangers de \#développement, affirme le président Xi. Le parti communiste chinois lutte pour le bien-être des Chinois et le développement humain" (12/01/2017 19:30:12@ChinaEUMission). Ce tweet illustre bien le parti pris des leaders du parti communiste de valoriser l'essor pacifique de la Chine dans le but de faire taire les craintes exprimées par les journalistes de la plupart des médias internationaux. Dans un contexte international marqué par le protectionnisme commercial et par l'expansion économique et géopolitique de la Chine à travers la " nouvelle route de la soie », le gouvernement chinois a souvent utilisé twitter pour expliquer sa politique économique : « l'ouverture de la Chine n'est pas un jeu à somme nulle, mais une coopération gagnantegagnante pour le monde. - dit le président Xi»(31/10/2017 16:56:57@ChinaEUMission); «RT@cgtnamerica: 'La mondialisation n'est pas un monstre redoutable', a déclaré le ministre des affaires étrangères chinois lors de la \#MunichSecurityConference » (20/02/2017 19:23:11@ChinaEmbOttawa, retweet du Twitter de CGTN, la télévision officielle chinoise en langue étrangère).

Les comptes Twitter de diplomates chinois sont le reflet de la politique officielle, les voix individuelles ne s'y expriment pas. En outre, ils traitent de problèmes sensibles, comme ceux liés au Tibet, et tentent d'imposer leur propre vision de l'occupation du Tibet par la Chine. L'ambassade de Chine à Ottawa a, par exemple, partagé deux vidéos intitulées « This is Tibet » sur Twitter le 9 et le 15 août 2017. Ces dernières racontent et montrent les grands changements économiques et sociaux qui ont marqué l'histoire récente du Tibet en s'appuyant sur une description concrète et incarnée des évolutions du mode de vie des Tibétains. Les films courts valorisent des politiques précises de développement mises en œuvre par le gouvernement chinois dans la région. Ce storytelling vise à transformer l'envahisseur en un bienfaiteur dans une dialectique rhétorique très classique du colonialisme. De nombreux Tibétains prennent la parole dans ces films, mais cette polyphonie n'est pas synonyme de diversité des voix car celles qui ont été sélectionnées pour s'exprimer servent sans aucune nuance la communication diplomatique de la Chine.

\section{Construire une image de puissance mondiale de premier ordre}

En tant que puissance politique, la Chine participe de manière accrue, confiante et efficace à la gouvernance mondiale. Cette montée en puissance inquiète et suppose un discours de réassurance sur le caractère pacifique de cet essor. 182 tweets publiés par les ambassades chinoises peuvent être analysés comme tournés vers la valorisation de la Chine en tant que pays responsable et soucieux de participer à la gouvernance internationale, d'être exemplaire en termes d'actions caritatives internationales, de lutte contre la pauvreté, mais aussi le changement climatique, la désertification, l'exploitation des ressources naturelles.

Bien que la quasi-totalité des tweets analysés exprime des positions dans la droite ligne de la voix diplomatique officielle, les diplomates chinois tentent de fabriquer de la diversité polyphonique à travers la pratique de plus en plus assumée de retweets des messages publiés par des comptes officiels d'ONG et de leaders d'opinion. Ces messages présentent un point commun qui est de rendre compte d'informations valorisantes pour la Chine et son gouvernement. Ainsi, les comptes Twitter diplomatiques chinois interagissent régulièrement avec ceux d'organisations internationales et de diplomates étrangers sur les questions de changement climatique : «RT@ GreenpeaceEAsia: Bonne nouvelle! La Chine a déjà réalisé son objectif d'énergie solaire de 2020»(08/28/2017 11:55:02@ChinaEUMission); «Le 
rapport@IEA montre \#China est le plus grand investisseur et créateur en matière d'énergies propres du monde, la Chine montre la voix en matière de lutte contre \#climatechange» (07/12/2017 13:59:17@ChinaEUMission) ; «@ErikSolheim: la plus grande ferme solaire flottante au monde a commencé à produire de l'électricité à Huainan, dans la province de l'Anhui, en Chine orientale»(17/08/2017 12:33:18@ChinaEUMission).

\section{Promouvoir la Chine dans une optique de soft power}

L'amélioration de l'image des États-nations est un objectif central de la diplomatie publique, auquel les comptes Twitter diplomatiques chinois participent. Au moins de 342 tweets illustrent les efforts des diplomates chinois pour valoriser la culture de leur pays. Ces tweets visent à 1) rendre compte du développement économique du pays et de ses progrès en terme social ; 2) promouvoir des innovations scientifiques et technologiques (comme le TGV chinois, l'e-commerce); 3) raconter la Chine dans un storytellling subtil qui exploite les mythes, les héros, les symboles de la culture chinoise : les 24 fêtes correspondant 24 termes solaires, les festivals chinois, les symboles chinois comme le dragon ou encore animal mascotte, le panda ; 4) montrer des images et des vidéos qui permettent de ré-humaniser la Chine à travers des images de lieux habités, de pratiques culturelles ou de coutumes chinoises dans leur dimension éventuellement folklorique.

La fonction « retweet 》 confère à ces comptes diplomatiques un verni polyphonique. Les ambassades chinoises citent régulièrement les recherches et découvertes d'universitaires, ainsi que les propos de dirigeants politiques étrangers quand ils sont susceptibles de valoriser la Chine. Ce retweet participe au soft power chinois à l'instar de la publication d'entretiens entre des diplomates chinois et différents dirigeants politiques internationaux qui ont une vision émerveillée du développement économique chinois. Parallèlement, les diplomates utilisent des tweets ou des retweets pour faire connaître les entreprises chinoises et leurs produits à un public international. Le 14 juillet 2017, le compte Twitter@ChinaEmbOttawa retweeta un message de Richard Liu, un chef d'entreprise chinoise en e-commerce, pour diffuser und nouvelle inattendue d'ouverture du marché chinois entreprises canadiennes : «chers commerçants canadiens : la Chine veut vos produits » (14/07/2017 23:27:32), ce tweet est dans le but de rappeler l'importance et l'opportunité des relations économiques et commerciales bilatérales entre la Chine et le Canada. 


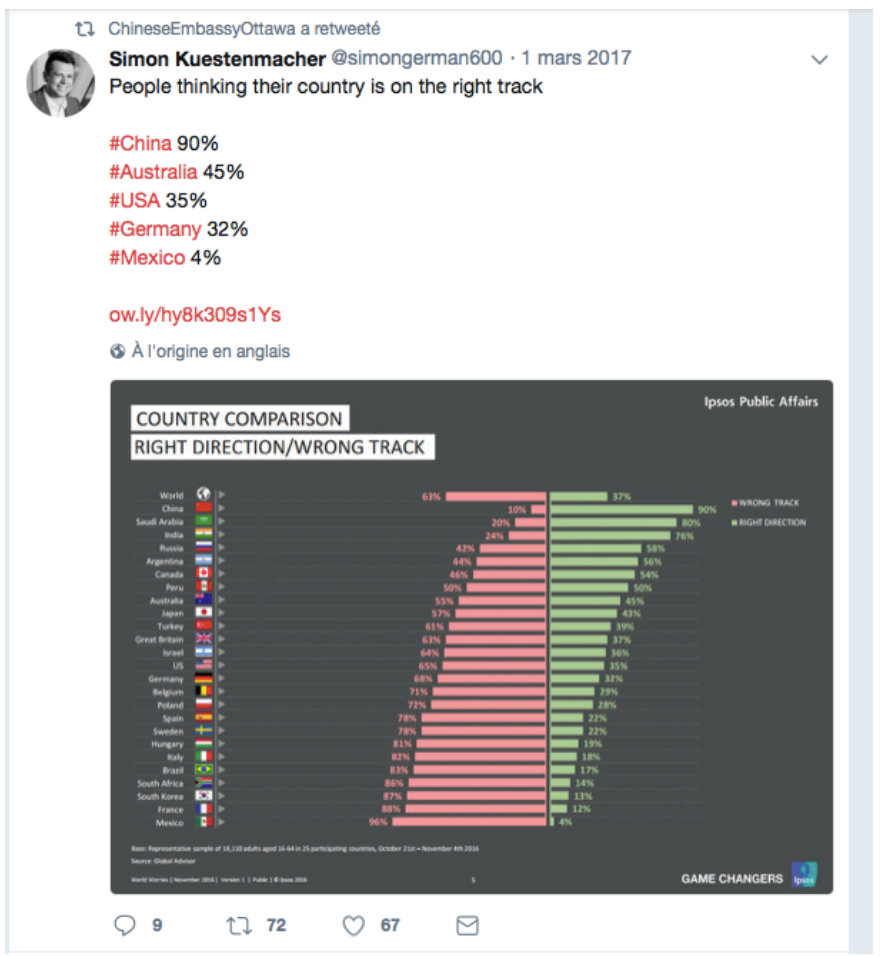

(Légende : illustration de la manière dont un compte diplomatique chinois se positionne en infomédiaire en retweetant des infographies qui valorisent la Chine)

\section{Une infomédiation génératrice d'une impression de polyphonie}

La diplomatie publique chinoise sur Twitter associe des messages qui illustrent l'ambiguïté de son positionnement qui doit à la fois respecter un certain nombre des codes de twitter pour espérer être suivie, pour que ses messages soient commentés et retweetés et qui doit pour autant s'inscrire dans un cadre très contraignant. D'une part, les diplomates tentent d'introduire des contenus variés et de faire entendre une forme de polyphonie pour dynamiser l'image de leur institution et leur pays, et prouver l'ouverture de la Chine, sa capacité à dialoguer avec le monde, mais d'autre part, les comptes des diplomates sont utilisés comme s'il s'agissait de comptes du services-presse du ministère des Affaires étrangères servant de relais de diffusion pour les communiqués officiels. Cette pratique est la plus évidente dans la pratique de la communication twiplomatique de la Chine au Canada (corpus de 350 tweets). Plus des trois quarts des tweets sont des informations officielles diplomatiques par lesquels la voix unique du gouvernement de Pékin s'exprime: les textes complets des discours des dirigeants (principalement le président chinois, le premier ministre, le ministre des Affaires étrangères et l'ambassadeur de Chine au Canada); les communiqués de presse des "deux sessions » plénières annuelles de l'Assemblée populaire nationale (APN) et du Comité national de la Conférence consultative politique du peuple chinois (CCPPC); les nouvelles sur les échanges bilatéraux; des messages liés à l'emploi du temps officiel de l'ambassadeur chinois au Canada. Ces comptes de diplomates servent cependant aussi beaucoup de relais de diffusion pour les dépêches des agences de presse nationales officielles de la Chine, notamment Xinhua, CGTN et le Quotidien du Peuple. Les comptes diplomatiques chinois de Bruxelles et Ottawa retweetent ainsi des articles et des dépêches sans les commenter, ils s'effacent en temps qu'infomédiateurs accréditant l'idée qu'une seule entité s'exprime derrière 
un verni polyphonique. Le concept d'infomédiation est ici utilisé au sens d'«autorité informationnelle [qui] n'a pas pour fonction principale l'influence, mais celle d'informer (donner une forme) » (Broudoux, 2017), il gomme donc la caractère politique et stratégique de l'activité qui consiste à citer une source extérieure et les dimensions réticulaires d'échange de bons procédés et de renforcement mutuel.

Pour autant, l'idée que le dispositif socio-médiatique de Twitter faciliterait les échanges entre utilisateurs est vérifiée pour ce qui concerne les diplomates chinois et leurs homologues étrangers. Dans 233 tweets, on observe des discussions au sens où les personnes en charge des comptes diplomatiques à l'origine des tweets répondent toujours aux commentaires. Ces échanges relèvent du registre de la conversation de l'échange de politesse, mais ils s'inscrivent les relations diplomatiques dans une forme de personnalisation et de convivialité. Début 2017, Ivanka Trump, fille du président américain Trump, publia une vidéo de sa fille en train de chanter des chansons traditionnelles chinoises pour exprimer ses salutations au peuple chinois pour le Nouvel An. L'ambassade de Chine à Ottawa retweeta ce message d'Ivanka Trump et ce retweet fut à son tour réexpédié 3.757 fois. Durant la période du Nouvel An chinois, l'ambassade de Chine à Ottawa interagit avec Justin Trudeau, le Premier ministre du Canada, sur Twitter à travers un échange de vœux pour la nouvelle année. Cet échangé a également été retransmis 1.389 fois. Les comptes Twitter des diplomates chinois interagissent en parallèle avec ceux des ministères étrangers, le compte de Twitter@ChinaEmbOttawa échange avec l'ambassade du Canada à Pékin pour célébrer les nouveaux records économiques et touristiques bilatéraux : " +610000 touristes chinois ont visité au Canada en 2016 et le nombre de visiteurs a augmenté ! \#CanadaChina, RT@CanadaChine: La Chine est le deuxième important partenaire commercial pour Canada et le troisième important marché en tourisme pour Canada » (10/27/2017 $18: 50: 28)$.

\section{Conclusion}

Les voix diplomatiques chinoises sur Twitter sont peu nombreuses et leurs discours sont en parfait unisson du fait du cadre politique très contraignant dans lequel elles s'expriment et conformément à l'éthos diplomatique. Pour autant, ces voix participent à l'évolution de la pratique diplomatique du gouvernement chinois d'un modèle purement propagandiste vers un modèle de diplomatie publique, polyphonique et capable d'articuler harmonieusement une grande diversité de registres, de contenus, en se présentant comme un infomédiaire de dépêches et d'articles d'agences de presse, ainsi que de communiqués officiels, mais aussi sur un mode mineur, mais non anecdotique des messages valorisant la Chine dans une perspective de soft power alors qu'ils n'ont pas été pensés comme tels : articles scientifiques publiés par des chercheurs occidentaux, vœux de dirigeants occidentaux. Consensuels et policés, les messages sur Twitter des comptes diplomatiques chinois à Bruxelles et Ottawa ont aussi intégré les règles d'usages propres aux médias sociaux numériques, comme la personnalisation, à travers la mise en valeur d'histoires et de visages, l'humour à travers des clins d'œil notamment lors des vœux, l'utilisation de la mascotte sympathique du panda et l'interactivité avec une systématisation des réponses aux commentaires.

L'analyse netnographique de tous les tweets publiés par les deux comptes Twitter chinois à Bruxelles et à Ottawa illustre les efforts de la diplomatie chinoise pour intégrer les règles tacites d'usage des médias sociaux et les rendre compatibles avec des objectifs 
stratégiques et une tradition de communication centralisée sous contrôle et purement descendante. Les tweets du corpus qui émanent directement des propriétaires des comptes relèvent de types de discours variés bien que les messages les plus institutionnels dominent. On y relève des messages très éloignés du champ politique, qui valorisent des performances économiques, des histoires individuelles ou des aspects folkloriques de la culture chinoise dans une perspective de marketing touristique. De plus, bien que le gouvernement chinois gère peu de comptes twiplomatiques, l'utilisation accrue de la fonction « retweet » par les diplomates confère un caractère de polyphonique à ses comptes et créent autour d'eux un réseau souple de sympathie à travers des échanges de politesse certes conventionnels (« merci pour le retweet! »). Cette fonction « retweet » multiplie les possibilités de conversations entre les organisations et leurs publics (Boyd, Golder, et Lotan, 2010, p. 10), peu importe que celles-ci soient superficielles ou développées, libres ou sous contrôle. Ces ferments de conversations favorisent l'établissement de relations entre le gouvernement chinois et ses nombreuses parties prenantes internationales qui sont présents sur les médias sociaux en même temps qu'ils contribuent à forger l'image d'une Chine ouverte au dialogue et capable de dialogue, moins austère et plus tolérante en matière de liberté d'expression. Pékin mobilise donc largement la fonction « retweet » de Twitter pour faire la promotion du pays au sens large (gouvernance, performance économique, implication en matière de responsabilité sociale et environnementale, qualité de vie, richesse du patrimoine culturel, sens de la fête, solidarité nationale, valeurs humanistes et caritatives) ce qui lui permet de conjurer la critique en propagande grossière et en autopromotion nombriliste. Les comptes diplomatiques chinois illustrent l'intelligence des stratégies de communication par délégation en laissant à d'autres le soin de présenter la Chine sous son meilleur jour. Pour autant, ces comptes diplomatiques qui sont la pointe visible des stratégies de soft power chinois restent très marqués par des mécanismes et des dispositifs de censure a priori, et par un écosystème politique brutal qui génère de l'autocensure. De fait, le gouvernement chinois sur Twitter s'exprime certes de manière polyphonique, mais dans un unisson quasi parfait, et cette harmonie est parfaitement stratégique eu égard aux buts visés de promotion du « rêve chinois ». Les diplomates du pays du panda se sont donc bien appropriés Twitter. Et, l'oiseau bleu ne fait pas que diffuser les discours officiels du plantigrade et renforcer son autorité (Merzeau, 2013), il l'aide aussi à développer son capital de sympathie, un atout non négligeable pour une stratégie de soft power conquérante.

\section{Référence :}

ARIFON, O., « Chine : de la muraille à Internet, permanence du contrôle étatique », Hermès, $\mathrm{n}^{\circ}$ 63, 2009, p. 160-164.

ARSÈNE, S., «Quand les internautes chinois prennent la parole : les nouveaux repères de l'identité », Hermès, $n^{\circ} 55,2009$, p. 17-22.

Arsène, S., « Protester sur le Web chinois (1994-2011) », Le Temps des médias, n 1, 2012, p. $99-110$.

Botan, C.H. et Taylor, M., " Public Relations: State of the Field », Journal of Communication, $\mathrm{n}^{\circ}$ 54, 2004, p. 645-661.

Boyd, D., Golder, S., et Lotan, G., " Tweet, tweet, retweet: Conversational aspects of retweeting on twitter», in $43^{\text {rd }}$ Hawaii International Conference on System Sciences, Honolulu, 2010, p. 1-10. 
BroudouX, E., « Construction de l'autorité informationnelle sur le web », HAL, https://archivesic.ccsd.cnrs.fr/file/index/docid/120710/filename/AutorInfo.pdf

CHEN, X.H. et LIU, X., « Les conceptions stratégiques des relations publiques étatiques sur la communication des valeurs fondamentales chinoises (notre traduction de chinois : Hexin jiazhiguan chuanbo de guojia gonggong guanxi zhanlüe gouxiang) », Modern Communication, $\mathrm{n}^{\circ} 227,2015$, p. 25-31.

D’hooghe, I., " Public diplomacy in the People's Republic of China », in Melissen, J. (dir.), The New Public Diplomacy: Soft power in International Relations, Basingstoke, Palgrave Macmillan, 2008, p. 88-105.

LAFRANCE, J.-P., « La révolution peut-elle être gazouillée ? », Hermès, no ${ }^{\circ}$ 61, 2011, p. 203211.

LE BELZIC, S., «Le nouveau rêve impérial de Xi Jinping », Valeurs actuelles, http://valeursactuelles.com/nouveau-r\%C3\%AAve-imp\%C3\%A9rial-xi-

jinping20130326.html, consulté le 29 mars 2018

LI, A. K., "Vers une méthode plus proactive - Le contrôle de l'opinion publique sur les microblogs chinois sous la nouvelle présidence de Xi Jinping », Perspectives chinoises, $\mathrm{n}^{\circ} 4$, 2015, p. 15-24.

Malone, G.D., « Managing Public Diplomacy », The Washington Quarterly, n 3, 1985, p. 199-213.

MERzeAU, L., "Twitter : une machine à fabriquer de l'autorité », dans Gabriel Gallezot, Nicolas Pélissier (dir.), Twitter, un monde en tout petit? , L'harmattan, 2013, p.35-52.

Muller, P., "Référentiel », in Boussaguet, L., Jacquot, S. et Ravinet, P. (dir.), Dictionnaire des politiques publiques 4e édition, Paris, Presses de Sciences Po, 2014, p. 555562.

OlLIVIER-YANIV, C., « De l'opposition entre "propagande" et "communication publique" à la définition de la politique du discours : proposition d'une catégorie analytique », Quaderni. Communication, technologies, pouvoir, $\mathrm{n}^{\circ} 72$, 2010, p. 87-99.

TuCH, H.N., Communicating with the world: U.S. public diplomacy overseas, New York, St. Martin's Press, 1990.

WANG, Y., " Public Diplomacy and the Rise of Chinese Soft Power », The ANNALS of the American Academy of Political and Social Science, $\mathrm{n}^{\circ}$ 1, 2008, p. 257-273.

WeICK, K.E., Sensemaking in organizations, Londres, Sage Publications, 1995.

ZAHARnA, R.S., Battles to Bridges, Londres, Palgrave Macmillan UK, 2010.

ZhONG, X. et LU, J. " Public diplomacy meets social media: A study of the U.S. Embassy's blogs and micro-blogs », Public Relations Review, n 5, 2013, p. 542-548.

ZHOU, Q.A., « Du changement de contexte international à la promotion des droits de parole: analyse sur les enjeux des relations publiques nationales de la Chine (notre traduction de chinois: cong guoji yujing bianqian dao huayuquan tisheng: shilun zhongguo guojia gongguan de jiyu yu tiaozhan)», News and Writing, $\mathrm{n}^{\circ}$ 10, 2010, p. 15-18. 